\title{
Characterization of Carbapenem-Resistant Acinetobacter baumannii Strains Isolated from Hospitalized Patients in Palestine
}

\author{
Regeen Handal, ${ }^{1,2}$ Lulu Qunibi, ${ }^{3}$ Ibrahim Sahouri, ${ }^{4}$ Maha Juhari, ${ }^{5}$ \\ Rula Dawodi, ${ }^{1}$ Hiyam Marzouqa, ${ }^{1}$ and Musa Hindiyeh ${ }^{1,2,6}$ \\ ${ }^{1}$ Caritas Baby Hospital, Bethlehem, State of Palestine \\ ${ }^{2}$ Bethlehem University, Bethlehem, State of Palestine \\ ${ }^{3}$ Alia Hospital, Hebron, State of Palestine \\ ${ }^{4}$ Hussein Hospital, Bethlehem, State of Palestine \\ ${ }^{5}$ Rafidia Hospital, Nablus, State of Palestine \\ ${ }^{6}$ Palestinian Forum for Medical Research, Ramallah, State of Palestine
}

Correspondence should be addressed to Musa Hindiyeh; mousa.hindiyeh@crb-mail.org

Received 25 March 2017; Accepted 18 June 2017; Published 26 July 2017

Academic Editor: Dulal Borthakur

Copyright $\odot 2017$ Regeen Handal et al. This is an open access article distributed under the Creative Commons Attribution License, which permits unrestricted use, distribution, and reproduction in any medium, provided the original work is properly cited.

\begin{abstract}
The American Centers for Disease Control and Prevention (CDC) recognizes Acinetobacter baumannii as a source of global outbreaks and epidemics especially due to its increasing resistance to commercially available antibiotics. In this study, 69 single patient multidrug resistant isolates collected from all over Palestine, except Gaza, were studied. All the isolates were resistant to all the $\beta$-lactam antibiotics including the carbapenems. Of the 69 isolates, $82.6 \%$ were positive for $b l a_{\text {OXA-23 }}, 14.5 \%$ were positive for $b l a_{\text {OXA-24 }}$, and $3 \%$ were positive for $b l a_{\text {OXA-58 }}$. None were positive for $b l a_{\text {OXA-143 }}$ and $b l a_{\text {OXA-235 }}$. In addition, $5.8 \%$ and $0 \%$ were positive for $b l a_{\mathrm{NDM}}$ and $b l a_{\mathrm{KPC}}$, respectively. Of the 69 isolates, none were positive for the aminoglycoside $a p h A 6$ gene while $93 \%$ were positive for the aphA1 gene. The acetyltransferases aacCl and aacA4 genes tested positive in $22 \%$ and $13 \%$ of the isolates, respectively. The ompA biofilm-producing virulence gene was detected in all isolates. Finally, Multilocus Sequence Typing (MLST) of 13 isolates revealed that more than one strain of A. baumannii was circulating in Palestinian hospitals as results revealed that 7 isolates were of ST208, 2 isolates ST218, 1 isolate ST231, 1 isolate ST348, and 2 new Sequence Types. The detection of these drug resistant pathogens is a reminder of the importance of active surveillance for resistant bacteria in order to prevent their spread in hospital settings.
\end{abstract}

\section{Introduction}

Acinetobacter baumannii is a gram-negative, nonfermentative, oxidase-negative coccobacillus. The Centers for Disease Control and Prevention (CDC) recognizes multidrug resistant (MDR) A. baumannii as a source of global outbreaks and epidemics especially due to its effectiveness in colonizing hospital environments and due to its increasing resistance to commercially available antibiotics, including $\beta$-lactams, fluoroquinolones, tetracyclines, and aminoglycosides [1-3]. A. baumannii is associated with hospital-acquired infections which include ventilator-associated pneumonia, bloodstream infections, meningitis, and urinary tract infections (UTI's) [3].

Mechanisms of resistance in Acinetobacter strains include efflux pumps, $\beta$-lactamases, and modifications in porin proteins. A. baumannii expresses aminoglycoside-modifying enzymes (AMEs) making them resistant to aminoglycoside antibiotics. Moreover, mutations in the gyrA and parC genes make them resistant to quinolones [1]. Worthy of mention are the findings of Fournier et al. (2006) who identified AbaR1 resistance islands ( $86 \mathrm{~kb}$ region) encompassing a cluster of resistance genes, namely, ones coding for tetracycline efflux pumps, several AMEs, AmpC, and OXA-10 $\beta$-lactamases. 
Genetic analysis of this region also indicated the presence of transposons and genes formerly identified in Salmonella spp. and E. coli [4].

Enzymatic degradation by $\beta$-lactamases is the most prevalent mechanism of $\beta$-lactam resistance in $A$. baumannii. Serine oxacillinases (Ambler class D OXA-type) and metallo$\beta$-lactamases (MBLs) (Ambler class B) are $\beta$-lactamases with carbapenemase activity. OXA-type carbapenemase genes in Acinetobacter can be classified into different phylogenetic subgroups: $b l a_{\mathrm{OXA}-23}, b l a_{\mathrm{OXA}-24}, b l a_{\mathrm{OXA}-51}$, $b l a_{\text {OXA-58 }}, b l a_{\text {OXA-143 }}$, and $b l a_{\text {OXA-235 }}[5,6]$. The emergence of carbapenem-resistant strains associated with OXA-type $\beta$-lactamases is increasing as well as their contribution to outbreaks and patient mortality [7]. Another group of recently discovered $\beta$-lactamases in Acinetobacter is the class B $\beta$-lactamase $b l a_{\mathrm{NDM}}$ and the class A serine carbapenemase $b l a_{\mathrm{KPC}}$ both have a great opportunity to spread to other bacteria due to their location on mobile genetic elements [8].

In addition, $A$. baumannii has other virulence factors that aid in its protection and survival. It has the ability to form biofilms, which are essentially aggregates in which the cells adhere to each other and to a surface in a self-produced matrix of extracellular DNA, polysaccharides and proteins [1]. In A. baumannii, outer membrane protein A (ompA) is involved in biofilm formation on abiotic surfaces [9]. Biofilm formation not only helps protect the bacteria against disinfection for instance, but also helps in trading resistance genes between the participating cells.

In this study, we performed in-depth characterization of extremely resistant nonduplicated $A$. baumannii strains isolated from samples of hospitalized patients from all over Palestine except Gaza.

\section{Materials and Methods}

2.1. Ethical Considerations. This study was approved by the Medical Research Committee at Caritas Baby Hospital (MRC-014) in Bethlehem, Palestine.

2.2. Study Population. Extremely drug resistant A. baumannii isolates, all resistant to carbapenems, were collected from 69 patients from Palestinian hospitals all over Palestine with the exception of the Gaza strip. Isolates were collected from five Palestinian districts as follows: Jerusalem $(N=2)$, Bethlehem $(N=22)$, Hebron $(N=34)$, Nablus $(N=8)$, and Ramallah $(N=3)$. The isolates were collected between January 2006 and February 2014 from blood, urine, sputum, and rectal samples.

2.3. A. baumannii Species Identification. In order to identify A. baumannii from the collected Acinetobacter spp. isolates, all isolates were identified biochemically and screened for the naturally occurring carbapenemase gene $\left(b l a_{\text {OXA-51 }}\right)$ intrinsic to $A$. baumannii using polymerase chain reaction (PCR) as previously reported $[10,11]$.

2.4. Presence of ISAba1 Element and the Expression of bla ${ }_{O X A-51}$ Gene. The isolates were screened for the presence of the IS element, ISAbal, and analyzed for the potential expression of the $b l a_{\text {OXA-51 }}$ gene by investigating the presence of the ISAbal element upstream of the gene [12].

2.5. Antibiotic Susceptibility Testing. Antibiotic susceptibility testing (AST) by disk diffusion test was performed for the following antibiotics (ceftazidime $30 \mu \mathrm{g}$, cefotaxime $30 \mu \mathrm{g}$, ceftriaxone $30 \mu \mathrm{g}$, piperacillin-tazobactam 100/10 $\mu \mathrm{g}$, cefepime $30 \mu \mathrm{g}$, sulfamethoxazole $25 \mu \mathrm{g}$, amikacin $30 \mu \mathrm{g}$, gentamycin $10 \mu \mathrm{g}$, ciprofloxacin $5 \mu \mathrm{g}$, meropenem $10 \mu \mathrm{g}$, and imipenem $10 \mu \mathrm{g})$. In addition, the minimal inhibitory concentration of the two antibiotics meropenem and colistin were determined by Etest (BioMérieux, Marcy-l'Étoile, France). AST was performed and interpreted according to the Clinical and Laboratory Standards Institute (CLSI) guidelines [13].

2.6. Detection of Antibiotics Resistant Genes and the BiofilmProducing ompA Gene. Carbapenem- and aminoglycosideresistant determinants as well as biofilm-producing virulence factors were screened for using standard PCR assay using a PTC-100 Peltier Thermal Cycler (Bio-Rad, USA). The PCR products were then subjected to gel electrophoresis on $1.5 \%$ agarose gel for 45 minutes at $85 \mathrm{~V}$ and viewed under UV light using the Transilluminator imaging device (Dinco \& Rhenium Industries Ltd., Israel). Target genes and their corresponding primers for PCR amplification are mentioned in Table 1. All PCR reactions were done in singleplex with the exception of the OXA-type class D carbapenemases $\left(b l a_{\text {OXA-23 }}, b l a_{\text {OXA-24 }}\right.$, and $\left.b l a_{\text {OXA-58 }}\right)$ which were done in a multiplex PCR reaction as reported in Mostachio et al. (2009). The assays were held in $25 \mu \mathrm{l}$ volume reactions containing 2x ReddyMix PCR Master Mix with $1.5 \mathrm{mM} \mathrm{MgCl}_{2}$ (Thermo Fisher Scientific, USA) with a primer concentration of $20 \mathrm{pmol} / \mu \mathrm{l}$ at standard PCR conditions. A PTC-100 Peltier Thermal Cycler (Bio-Rad, USA) was used for the PCR amplification reactions.

2.7. Multilocus Sequence Typing (MLST). A. baumannii bacterial DNA was extracted from 13 representative $A$. baumannii isolates using the High Pure Nucleic Acid Extraction Kit (Roche, Basel, Switzerland). The isolates covered the time period of the study (between 2006 and 2014) such that one isolate was from the year 2006, two were from the year 2007, one was from the year 2008, one was from the year 2010, two were from the year 2011, two were from the year 2012, and four isolates were from the year 2013. PCR reactions were performed for the seven housekeeping genes: gltA, gyrB, $g d h B, r e c A, c p n 60, g p i$, and $r p o D$ as previously reported by Bartual et al. (2005). PCR reactions were performed in $50 \mu \mathrm{l}$ volume reactions containing 2x ReddyMix PCR Master Mix with $1.5 \mathrm{mM} \mathrm{MgCl}_{2}$ (Thermo Fisher Scientific, USA) with a primer concentration of $20 \mathrm{pmol} / \mu \mathrm{l}$. The PCR conditions were those described by Bartual et al. with an annealing temperature of $55^{\circ} \mathrm{C}$ for all genes. A PTC-100 Peltier Thermal Cycler (Bio-Rad, USA) was used for the PCR amplification reactions. PCR products were analyzed on a $1.5 \%$ agarose gel and visualized using UV light using the Transilluminator imaging device (Dinco \& Rhenium Industries Ltd., Israel). Amplified PCR products with the appropriate size were purified using the High Pure PCR Product Purification 
TABLE 1: Summary of the target genes screened for using polymerase chain reaction (PCR).

\begin{tabular}{|c|c|c|c|c|}
\hline Target gene & Primer name & Sequence $\left(5^{\prime}\right.$ to $\left.3^{\prime}\right)$ & $\begin{array}{c}\text { Annealing } \\
\text { temperature }{ }^{*}\left({ }^{\circ} \mathrm{C}\right)\end{array}$ & $\begin{array}{l}\text { Amplicon size } \\
\text { (bp) }\end{array}$ \\
\hline \multirow{7}{*}{ Class D carbapenemases } & OXA-23 & $\begin{array}{l}\text { F: GATCGGATTGGAGAACCAGA } \\
\text { R: ATTTCTGACCGCATTTCCAT }\end{array}$ & 58 & 501 \\
\hline & OXA-24 & $\begin{array}{l}\text { F: GGTTAGTTGGCCCCCTTAAA } \\
\text { R: AGTTGAGCGAAAAGGGGATT }\end{array}$ & 58 & 246 \\
\hline & OXA-58 & $\begin{array}{l}\text { F: AAGTATTGGGGCTTGTGCTG } \\
\text { R: CCCCTCTGCGCTCTACATAC }\end{array}$ & 58 & 599 \\
\hline & \multirow{2}{*}{ OXA-51 } & F: TAATGCTTTGATCGGCCTTG & \multirow{2}{*}{58} & \multirow{2}{*}{353} \\
\hline & & R: TGGATTGCACTTCATCTTGG & & \\
\hline & OXA-143 & $\begin{array}{l}\text { F: TGGCACTTTCAGCAGTTCCT } \\
\text { R: TAATCTTGAGGGGGCCAACC }\end{array}$ & 58 & 180 \\
\hline & OXA-235 & $\begin{array}{l}\text { F: TTGTTGCCTTTACTTAGTTGC } \\
\text { R: CAAAATTTTAAGACGGATCG }\end{array}$ & 58 & 700 \\
\hline Class B carbapenemases & NDM & $\begin{array}{l}\text { F: CCAATATTATGCACCCGGTCG } \\
\text { R: ATGCGGGCCGTATGAGTGATTG }\end{array}$ & 58 & 812 \\
\hline Class A carbapenemases & KPC & $\begin{array}{l}\text { F: ATGTCACTGTATCGCCGTCT } \\
\text { R: TTTTCAGAGCCTTACTGCCC }\end{array}$ & 58 & 538 \\
\hline \multirow{6}{*}{ AME’s } & aphA6 & $\begin{array}{l}\text { F: ATGGAATTGCCCAATATTATTC } \\
\text { R: TCAATTCAATTCATCAAGTTTTA }\end{array}$ & 55 & 797 \\
\hline & aphA1 & $\begin{array}{l}\text { F: CAACGGGAAACGTCTTGCTC } \\
\text { R: ATTCGTGATTGCGCCTGAG }\end{array}$ & 58 & 455 \\
\hline & aacA4 & $\begin{array}{l}\text { F: ATGACTGAGCATGACCTTGCG } \\
\text { R: TTAGGCATCACTGCGTGTTCG }\end{array}$ & 65 & 518 \\
\hline & aadB & $\begin{array}{l}\text { F: ATGGACACAACGCAGGTCGC } \\
\text { R: TTAGGCCGCATATCGCGACC }\end{array}$ & 65 & 524 \\
\hline & aadA1 & $\begin{array}{l}\text { F: ATGAGGGAAGCGGTGATCG } \\
\text { R: TTATTTGCCGACTACCTTGGTG }\end{array}$ & 65 & 254 \\
\hline & aacC1 & $\begin{array}{l}\text { F: ATGGGCATCATTCGCACATGTAGG } \\
\text { R: TTAGGTGGCGGTACTTGGGTC }\end{array}$ & 65 & 456 \\
\hline Biofilm-nr & ompA & $\begin{array}{l}\text { F: CGCTTCTGCTGGTGCTGAAT } \\
\text { R: CGTGCAGTAGCGTTAGGGTA }\end{array}$ & 58 & 531 \\
\hline Blonim-procucing virulence ractors & epsA & $\begin{array}{l}\text { F: AGCAAGTGGTTATCCAATCG } \\
\text { R: ACCAGACTCACCCATTACAT }\end{array}$ & 58 & 451 \\
\hline
\end{tabular}

${ }^{*}$ Annealing temperature $\left({ }^{\circ} \mathrm{C}\right)$ used in this study.

Kit (Roche) and sequenced using the BigDye ${ }^{\circledR}$ Terminator v3.1 Cycle Sequencing Kit (Life Technology, Carlsbad, USA). The sequences were analyzed and cleaned using Sequencher ${ }^{\circledR}$ software (Gene Codes, MI, USA) and the PubMLST database. Isolates were assigned sequence types (STs) as per the allelic profiles in the A. baumannii MLST database (https://pubmlst.org/abaumannii/). The eBURST V3 algorithm (http://eburst.mlst.net/) was used to show phylogenetic relationships among related STs.

\section{Results}

3.1. Acinetobacter Species Identification. bla ${ }_{\mathrm{OXA}-51}$ PCR amplification confirmed that all 69 isolates were A. baumannii.

3.2. Presence of ISAba1 Element and the Expression of bla $a_{\mathrm{OXA}-51}$ Gene. Of the 69 isolates, 68 (99\%) carried the ISAbal element. Of these, only $30(43.5 \%)$ had the IS element upstream of the $b l a_{\text {OXA-51 }}$ gene.
3.3. A. baumannii Susceptibility Testing. All isolates had a multidrug resistant profile, and they were all resistant to all $\beta$ lactam antibiotics including the carbapenems (Figure 1). The carbapenem resistance profile of all isolates was confirmed by determining the MIC value of meropenem by Etest method (BioMérieux, Marcy-l'Étoile, France). The only class of antibiotics to which the isolates were susceptible was colistin sulfate (100\%) (Figure 1). In addition, the majority of A. baumannii isolates $(>90 \%)$ were resistant to the aminoglycosides, macrolides, and cotrimoxazole.

\subsection{Molecular Characterization}

3.4.1. Detection of Carbapenem-Resistant Genes. Of the 69 A. baumannii isolates tested, $57(82.6 \%)$ were positive for $b l a_{\text {OXA-23 }}, 10(14.5 \%)$ tested positive for $b l a_{\text {OXA-24 }}$, and $2(3 \%)$ tested positive for $b l a_{\text {OXA-58 }}$ (Figure 2). None of the isolates were positive for the class D $\beta$-lactamases $b l a_{\text {OXA-143 }}$ and $b l a_{\mathrm{OXA}-235}$. In addition, $3(5.8 \%)$ tested positive for the class $\mathrm{B}$ carbapenemase $b l a_{\mathrm{NDM}}$. Sequencing analysis of the $b l a_{\mathrm{NDM}}$ 


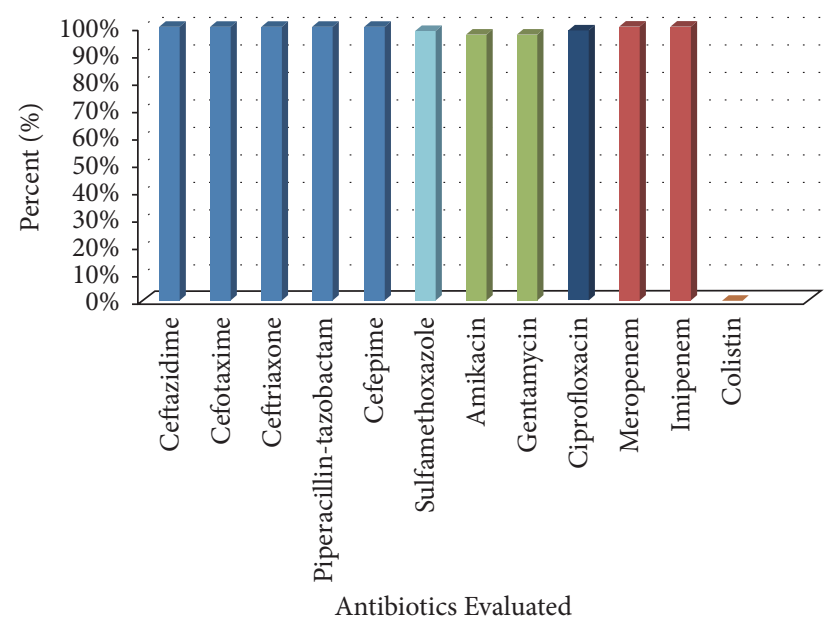

FIGURE 1: Histogram showing the percent resistance of Acinetobacter isolates to each antibiotic tested according to the CLSI guidelines.

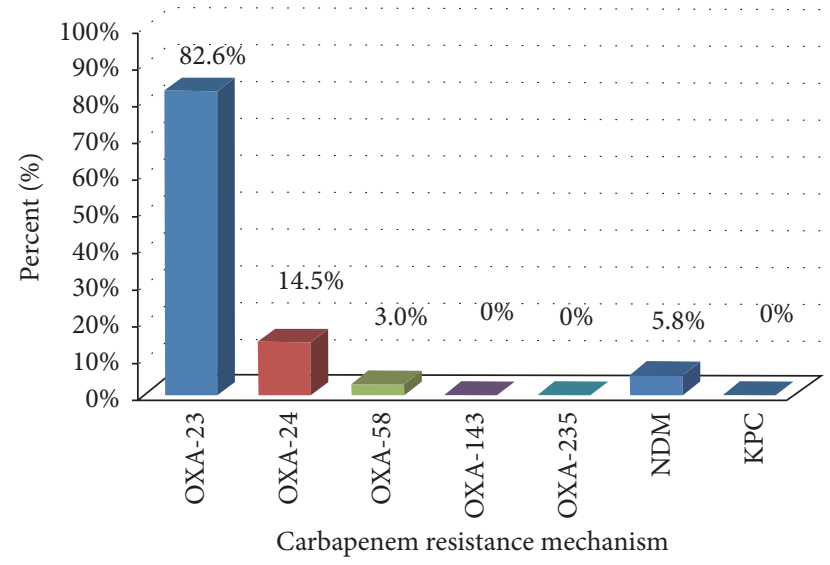

Figure 2: Histogram showing the distribution of carbapenemases in the Acinetobacter isolates tested.

gene revealed that the three isolates carried the $b l a_{\mathrm{NDM}-1}$ gene. None were positive for the class A carbapenemase $b l a_{\mathrm{KPC}}$. It is worthy to note that one MDR A. baumannii isolate was a combination OXA-23/OXA-24.

3.4.2. Detection of Aminoglycoside-Resistant Genes. Of the 69 isolates, 64 (93\%) were positive for the aphA1 gene while none were positive for the aphA6 gene (Figure 3). On the other hand, the isolates were positive for the acetyltransferases aac $\mathrm{C} 1$ and aacA 4 genes in 15 (22\%) and 9 (13\%) of the isolates, respectively. None were positive for the nucleotidyltransferases $a a d B$ or aadA1 genes (Figure 3).

3.4.3. Detection of Biofilm-Producing Virulence Factors. Of the 69 A. baumannii isolates tested, all (100\%) tested positive for ompA gene.

3.4.4. Multilocus Sequence Typing (MLST). Of the 13 A. baumannii strains evaluated by MLST, the following Sequence Types (STs) were obtained: ST208, ST218, ST231, ST348, and

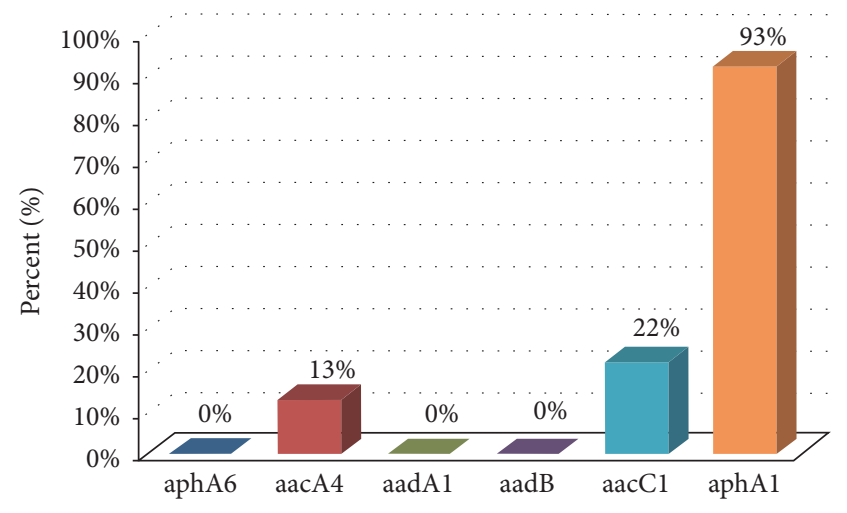

FIGURE 3: Histogram showing the distribution of AME's genes in the Acinetobacter isolates tested.

two new Sequence Types suggesting that more than one clone is circulating Palestinian hospitals. Two isolates did not have an exact ST match in the database. The nearest ST match yielded three and four possible STs for the A. baumannii isolates $\mathrm{CBH}|4248| 2007$ and $\mathrm{CBH} \backslash 2 \mathrm{D} 2 \backslash 2013$, respectively. After assigning sequence types for each isolate, phylogenetic relationships among related STs were established using the eBURST V3 algorithm (http://eburst.mlst.net/), and a dendrogram was generated (Figure 4). The central primary founder is ST208 [CBH|152|2012] (bootstrap = 1000). There were 13 linked single locus variants (SLVs) of the founder (ST75, ST544, ST138, ST21, ST3, ST584, ST467, ST218, ST92, ST238, ST69, CBH|2D2|2013, and ST533). Six of the SLVs of the founder (ST533, ST218, ST238, ST584, ST21, and ST3) have diversified to produce double locus variants (DLVs). There were a total of five subgroup founders (ST533, ST1, ST187, ST109, and ST231 [CBH|3750|2007]) that diversified and produced their own SLVs. One of the new STs [CBH|2D2|2013] has only one out of seven of the MLST loci altered when compared to the primary founder genotype, ST208. Its allelic profile has a single locus variant (SLV) from that of the founder's, ST208. On the other hand, the other new ST [CBH|4248|2007] has two out of seven MLST loci altered in comparison to the founder ST208. Hence, its allelic profile has a double locus variant (DLV) from that of the founder's.

\section{Discussion}

A. baumannii has acquired a huge genetic repertoire via horizontal gene transfer that makes it virulent and resistant to any environmental pressures $[1,4]$. Antibiotic susceptibility testing in this study showed that all $A$. baumannii isolates were resistant to the commercially available antibiotics with the exception of colistin. All isolates had an extremely drug resistant profile and they were resistant to carbapenems. MDR $A$. baumannii outbreaks were reported in hospitals worldwide including the Middle East $[14,15]$ and this is consistent with the increase in the incidence of health care associated A. baumannii infections reported in Palestinian and Israeli hospitals $[2,14,15]$. A. baumannii isolates from other geographical regions had a similar antimicrobial profile [16]. MIC tests also showed a similar result since most isolates were 


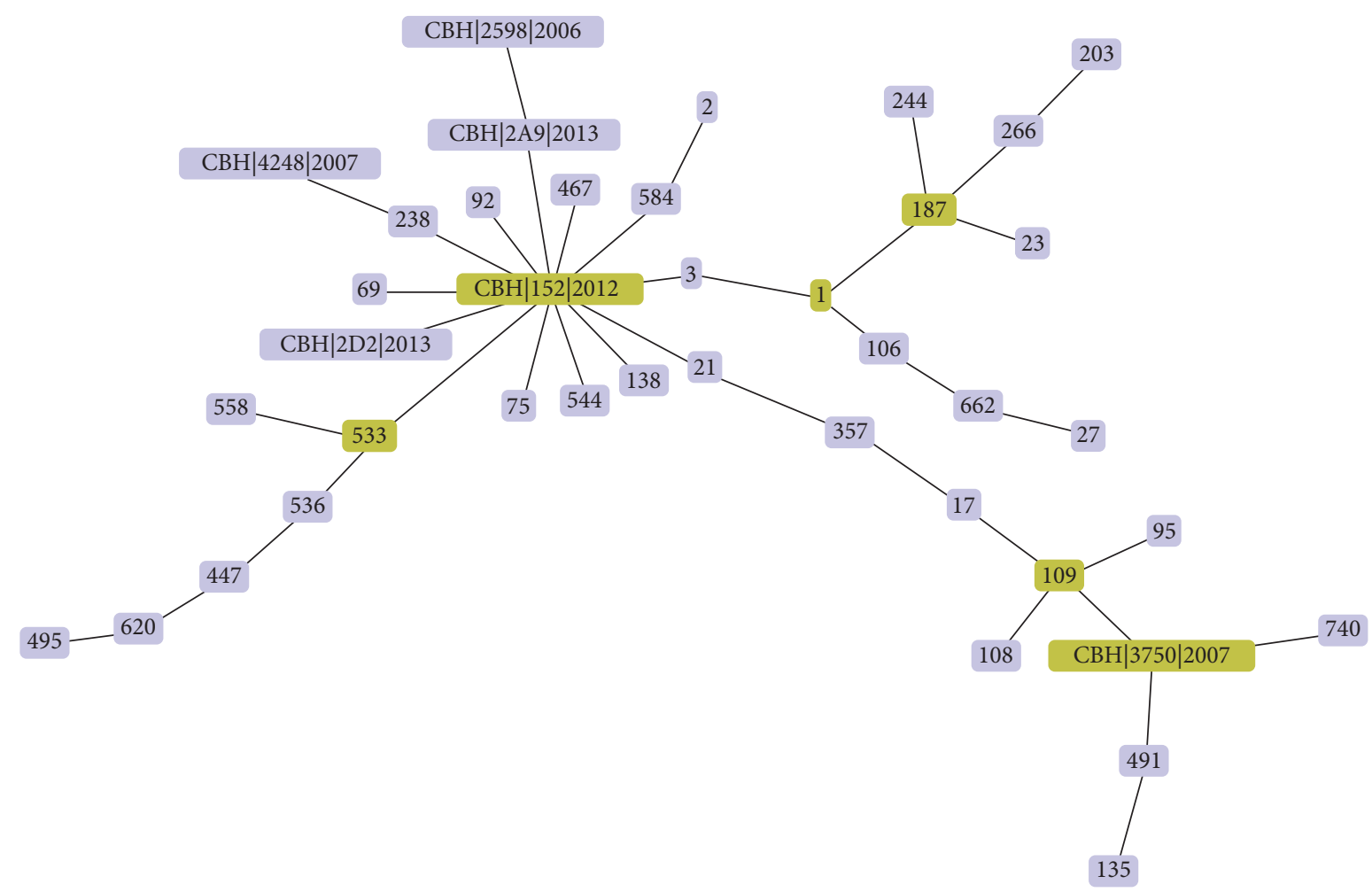

FIgURE 4: Population snapshot of the 13 A. baumannii isolates and existing reference isolates obtained from the PubMLST database, represented by the eBURST algorithm (http://eburst.mlst.net/). CBH|152|2012 (ST208) represents the primary founder with a bootstrap value = 1000, where CBH|2598|2006: ST348, CBH|4248|2007: New proposed ST, CBH|3750|2007: ST231, CBH|2A9|2013: ST218, CBH|152|2012: ST208, CBH|2D2|2013: New proposed ST.

resistant to meropenem and sensitive to colistin. There are, however, A. baumannii isolates that are becoming resistant to colistin [3].

Studying the carbapenem-resistant mechanisms of this pathogen was necessary in order to better control the spread of A. baumannii and devise a better antibiotics treatment plan. Carbapenem resistance was mainly attributed to the presence of $b l a_{\mathrm{OXA}}$ genes that produce carbapenemhydrolyzing enzymes [5]. Since the $b l a_{\text {OXA-51 }}$ gene is an oxacillinase naturally occurring mainly in A. baumannii, it was present in all $A$. baumannii isolates tested, and the potential expression of this gene in $43.5 \%$ of the isolates is facilitated by the ISAbal element present upstream of the gene. The production of $b l a_{\mathrm{OXA}-23}$ by an $A$. baumannii strain is enough to confer resistance to the carbapenems, and $82.6 \%$ of the isolates in this study carried this gene. This gene is plasmid-borne suggesting the mobility of this genetic segment facilitating horizontal gene transfer [5]. bla $a_{\text {OXA-23 }}$ was also reported in neighboring countries including Iraq, Greece, Italy, and Turkey [16]. Molecular class D $\beta$-lactamases confer resistance to the carbapenems and narrow-spectrum cephalosporins. $14.5 \%$ of the isolates also carried the $b l a_{\mathrm{OXA}-24}$ gene, and $3 \%$ of them carried the recently identified $b l a_{\text {OXA-58 }}$ gene. This is also the case in neighboring countries as those class $\mathrm{D} \beta$-lactamases have been reported in Iraq, Kuwait, Lebanon, Turkey, Italy, Greece, and the UK [16]. Enzymatic degradation by $\beta$-lactamases is the most prevalent mechanism of $\beta$-lactam resistance in A. baumannii [7], and this is clearly shown in our sampled $A$. baumannii isolates in the West Bank.

About $6 \%$ of our A. baumannii isolates tested positive for the class $\mathrm{B}$ carbapenemase $b l a_{\mathrm{NDM}-1}$. This is consistent with regional and global results as there are reports in Israel, India, China, United Kingdom, Canada, France, Sweden, Morocco, South Africa, United Arab Emirates, and Iran among many others [8].

The multidrug resistant profile of the isolates was also heightened by the presence of aminoglycoside-resistant determinants, namely, the phosphotransferase $a p h A 1$ and the acetyltransferases aacC1 and aacA4. Almost all our isolates carried the $a p h A 1$ gene, and $22 \%$ and $13 \%$ were positive for $a a c C 1$ and $a a c A 4$ genes, respectively. In comparison to other studies, Aliakbarzade et al. (2014) reported the following aminoglycoside-resistant genes in Iran: aacC1, aadA1, aphA6, and $a a d B$. Only aacC1 was detected in our Palestinian isolates, and none of our isolates carried the phosphotransferase aphA6. The aminoglycoside-resistant gene aphA6 was reported in Egypt [17]. On a more international scale, the following aminoglycoside-resistant genes were reported in Poland in 2013: aphA1, aphA6, and aacC1 [18]. Our isolates are equipped with not only enzymatic resistance mechanisms, but also the ompA biofilm-producing virulence factor. It is 
unfortunate that our A. baumannii isolates in the West Bank produce biofilms: $100 \%$ tested positive for ompA.

There is an apparent global outbreak of carbapenemresistant $A$. baumannii especially in the last decade. MLST is a discriminative typing method whose data are in concordance with other typing results produced by PFGE and AFLP analysis [5]. We sequence typed 13 isolates that are representative samples from different areas and different time periods throughout the West Bank. Sequence analysis of Palestinian genotypes showed a similarity with the global genotypes. Our MLST results reveal that more than one strain of A. baumannii is circulating in our Palestinian hospitals; the resulting STs were ST208, ST218, ST231, ST348, and two new STs. We correlated the STs with the timeline of their isolation and preliminary results show that there is a successful spread of clone. To illustrate, ST208 is evident throughout the studied time period, starting in 2008 up till 2014 , indicating a successful spread of that particular strain. Internationally, ST208 was reported in the US in 2009, then in Egypt the following year, and then in China in 2012 [19]. Interestingly, the seven ST208 isolates came from the same Palestinian geographical district, Hebron. There are reports of ST218 in the PubMLST database in the year 2000. But, locally in Palestine, our MLST results show that the two isolates, isolated in 2013 from urine and sputum samples, were of ST218. ST231 was first reported in Brazil in 2000 and later again in 2007 in the same country [19]. Our results report ST231 that belongs to a 2007 isolate. Internationally, there are reports of ST348 in the US in 2008 according to the PubMLST database. But this particular ST was detected in Palestine earlier in 2006 as per our MLST results. Worthy of mention is the fact that the isolates carrying the $b l a_{\mathrm{NDM}-1}$ gene did not belong to the same clone.

Moreover, new strains are emerging. According to our MLST results, there is a new strain that emerged in 2007, and another new one in 2013. Over time, the frequent use of antibiotics will result in the emergence of new resistant strains of $A$. baumannii bacteria.

The eBURST V3 algorithm [20] shows the relationship between the closely related isolates in which the founding genotype starts to diversify producing a cluster of closely related genotypes as it increases in frequency in the population. In summary, there is more than one clone of A. baumannii circulating worldwide as shown by the sequence analysis of our Palestinian genotypes and the global genotypes.

Resistance to antibiotics, resistance to disinfectants, and resistance to desiccation accompanied by the presence of drug resistant and biofilm-producing genes all contribute to the persistence of $A$. baumannii as a hospital pathogen. Resistant $A$. baumannii strains have a selective advantage in environments like the ICU's where antimicrobials and surface disinfectants are extensively used. Selection for resistant forms can take place during antimicrobial treatment or after treatment. There is a sense of altered microbial ecology with regard to resistant and susceptible bacteria as well as the types of microorganisms surviving after treatment.

Epidemiologically, A. baumannii is of concern due to its dissemination mostly in a clonal fashion whether it is within health care institutions and cities or globally between countries. Since A. baumannii is easily transmitted and can, therefore, cause outbreaks, certain control measures have to be taken into account. To start with, health care facilities and personnel should follow the CDC's infection control guidelines and protocols, which attempt to control and prevent the transmission of MDR organisms, such as active surveillance, hand hygiene, and contact precautions [19]. Molecular epidemiological studies should be implemented to determine the presence or absence of a clonal outbreak strain such as A. baumannii ST208 which appears to be a very successful clone. Proper environmental cleaning of potential reservoir sources present in hospital environments such as ventilators, catheters, containers, and moist articles is highly recommended to prevent the spread of Acinetobacter. Moreover, antibiotic stewardship programs must be initiated in order to properly manage treatment and spread of this deadly pathogen.

\section{Conflicts of Interest}

The authors declare that there are no conflicts of interest regarding the publication of this paper.

\section{References}

[1] L. S. Munoz-Price and R. A. Weinstein, "Acinetobacter infection," The New England Journal of Medicine, vol. 358, no. 12, pp. 1214-1281, 2008.

[2] F. Perez, A. M. Hujer, K. M. Hujer, B. K. Decker, P. N. Rather, and R. A. Bonomo, "Global challenge of multidrugresistant Acinetobacter baumanni," Antimicrobial Agents and Chemotherapy, vol. 51, no. 10, pp. 3471-3484, 2007.

[3] Y. Cai, D. Chai, R. Wang, B. Liang, and N. Bai, "Colistin resistance of Acinetobacter baumannii: clinical reports, mechanisms and antimicrobial strategies," Journal of Antimicrobial Chemotherapy, vol. 67, no. 7, pp. 1607-1615, 2012.

[4] P.-E. Fournier, D. Vallenet, V. Barbe et al., "Comparative genomics of multidrug resistance in Acinetobacter baumannii," PLoS Genetics, vol. 2, no. 1, 2006.

[5] A. Y. Peleg, H. Seifert, and D. L. Paterson, "Acinetobacter baumannii: emergence of a successful pathogen," Clinical Microbiology Reviews, vol. 21, no. 3, pp. 538-582, 2008.

[6] P. G. Higgins, F. J. Pérez-Llarena, E. Zander, A. Fernández, G. Bou, and H. Seifert, "OXA-235, a novel class D $\beta$-lactamase involved in resistance to carbapenems in Acinetobacter baumannii," Antimicrobial Agents and Chemotherapy, vol. 57, no. 5, pp. 2121-2126, 2013.

[7] S. Brown and S. Amyes, "OXA $\beta$-lactamases in Acinetobacter: the story so far," Journal of Antimicrobial Chemotherapy, vol. 57, no. 1, pp. 1-3, 2006.

[8] M. Berrazeg, S. Diene, L. Medjahed et al., "New Delhi metallobeta-lactamase around the world: an ereview using Google Maps," Eurosurveillance, vol. 19, no. 20, article 20809, 2014.

[9] A. H. Delcour, "Outer membrane permeability and antibiotic resistance," Biochim Biophys Acta, vol. 1794, no. 5, pp. 808-816, 2009. 
[10] A. K. Mostachio, I. Van Der Heidjen, F. Rossi, A. S. Levin, and S. F. Costa, "Multiplex PCR for rapid detection of genes encoding oxacillinases and metallo- $\beta$-lactamases in carbapenemresistant Acinetobacter spp," Journal of Medical Microbiology, vol. 58, no. 11, pp. 1522-1524, 2009.

[11] B. A. Evans and S. G. B. Amyes, “OXA $\beta$-lactamases," Clinical Microbiology Reviews, vol. 27, no. 2, pp. 241-263, 2014.

[12] J. F. Turton, M. E. Ward, N. Woodford et al., "The role of ISAba1 in expression of OXA carbapenemase genes in Acinetobacter baumannii," FEMS Microbiology Letters, vol. 258, no. 1, pp. 7277, 2006.

[13] Clinical and Laboratory Standards Institute (CLSI), "Performance Standards for Antimicrobial Susceptibility," January 2014, http://ncipd.org/control/images/NCIPD_docs/CLSI _M100-S24.pdf.

[14] A. M. Al Jarousha, A. H. El Jadba, A. S. Al Afifi, and I. A. El Qouga, "Acinetobacter baumannii infection in the neonatal intensive care unit," Iranian Journal of Public Health, vol. 37, no. 3, pp. 107-112, 2008.

[15] A. Abbo, S. Navon-Venezia, O. Hammer-Muntz, T. Krichali, Y. Siegman-Igra, and Y. Carmeli, "Multidrug-resistant Acinetobacter baumannii," Emerging Infectious Diseases, vol. 11, no. 1, pp. 22-29, 2005.

[16] R. Zarrilli, M. Crispino, M. Bagattini et al., "Molecular epidemiology of sequential outbreaks of Acinetobacter baumannii in an intensive care unit shows the emergence of carbapenem resistance," Journal of Clinical Microbiology, vol. 42, no. 3, pp. 946-953, 2004.

[17] M. El-Sayed-Ahmed, M. A. Amin, W. M. Tawakol, L. Loucif, S. Bakour, and J.-M. Rolain, "High prevalence of $b l a_{N D M-1}$ carbapenemase-encoding gene and 16S rRNA armA methyltransferase gene among Acinetobacter baumannii clinical isolates in Egypt," Antimicrobial Agents and Chemotherapy, vol. 59, no. 6, pp. 3602-3605, 2015.

[18] P. Nowak, P. M. Paluchowska, and A. Budak, "Co-occurrence of carbapenem and aminoglycoside resistance genes among multidrug-resistant clinical isolates of Acinetobacter baumannii from Cracow, Poland," Medical science monitor basic research, vol. 20, pp. 9-14, 2014.

[19] C. Camp and O. L. Tatum, "A review of Acinetobacter baumannii as a highly successful pathogen in times of war," Laboratory Medicine, vol. 41, no. 11, pp. 649-657, 2010.

[20] eBURST V3, August 2014, Available from http://eburst.mlst.net. 

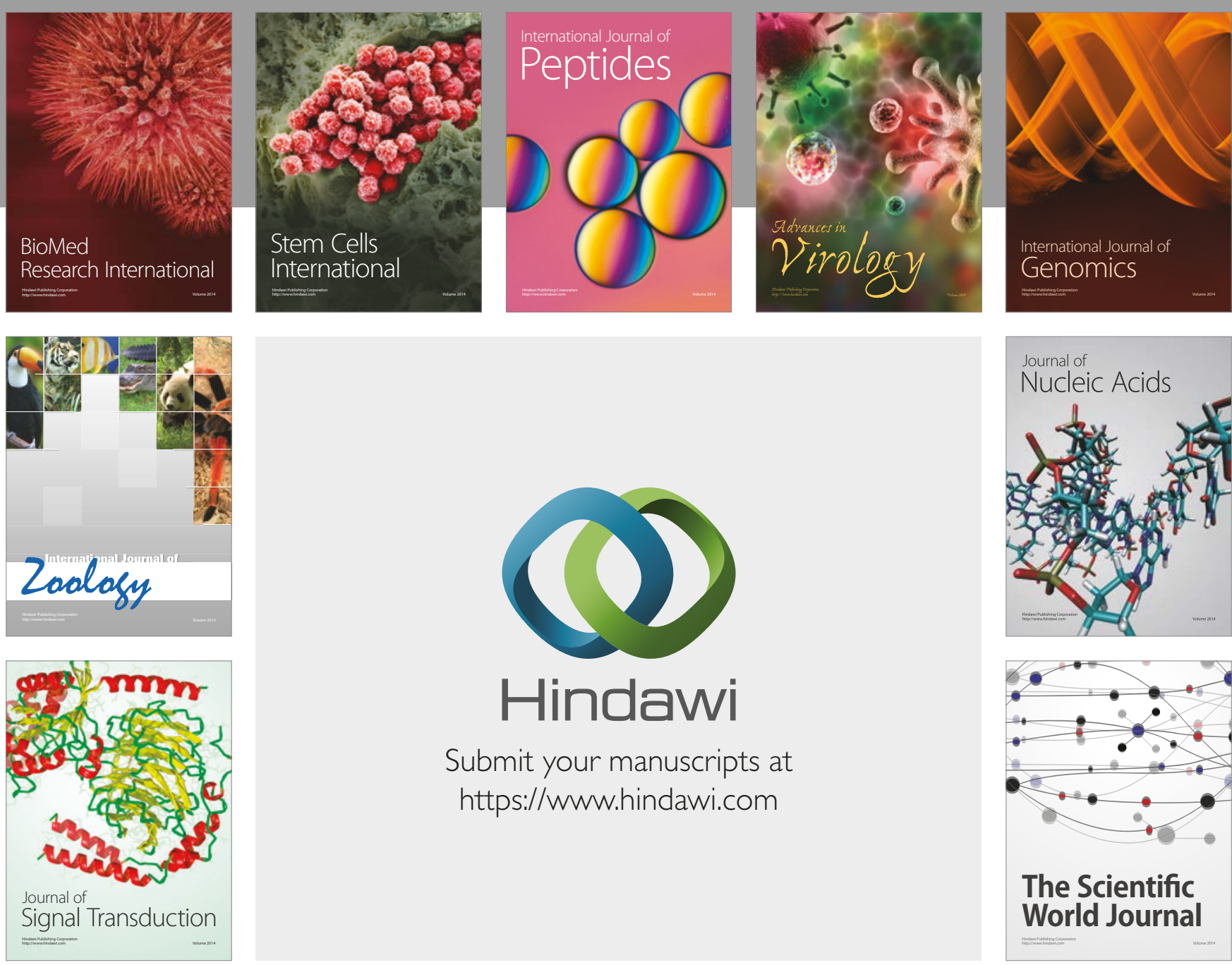

Submit your manuscripts at

https://www.hindawi.com
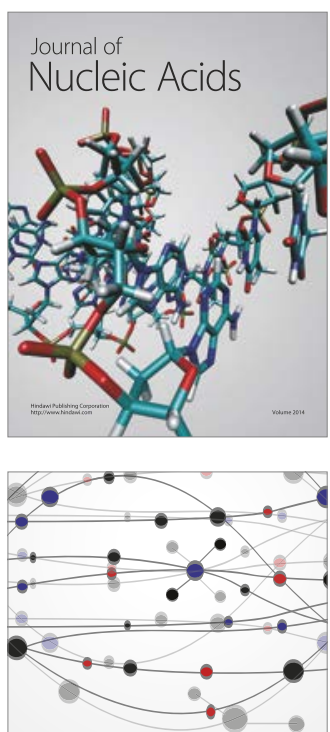

The Scientific World Journal

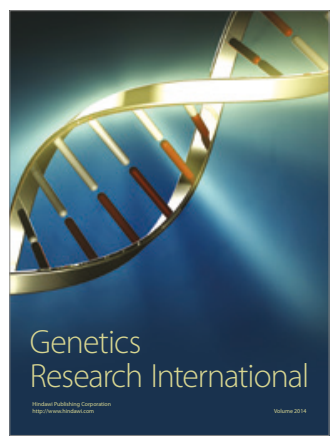

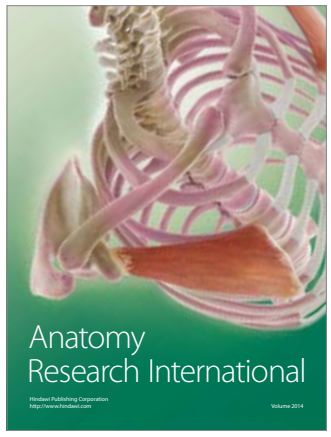

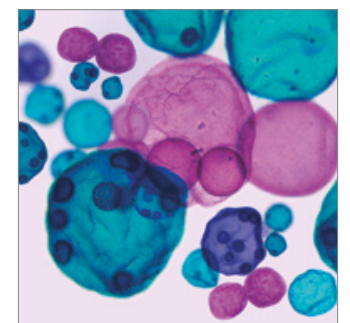

International Journal of Microbiology
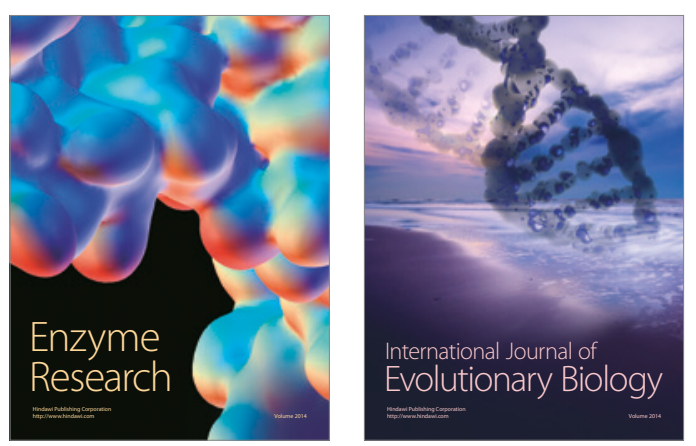
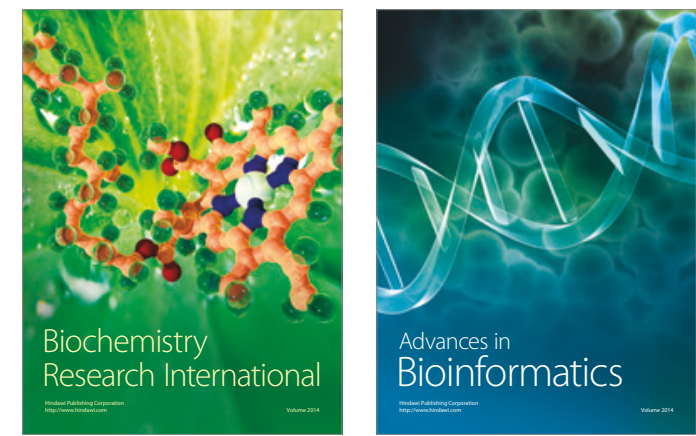

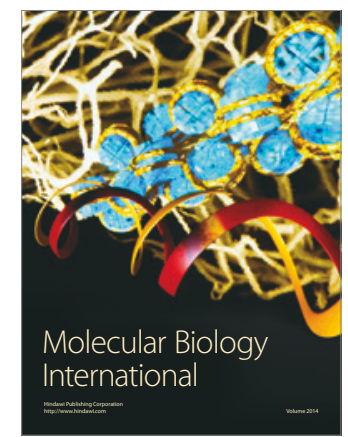

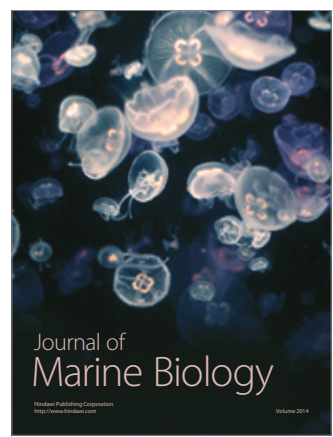

